\title{
Detecting racism and xenophobia using deep learning models on Twitter data: CNN, LSTM and BERT
}

\author{
José Alberto Benítez-Andrades ${ }^{\text {Corresp., } 1}$, Álvaro González-Jiménez ${ }^{2}$, Álvaro López-Brea ${ }^{2}$, Jose Aveleira-Mata ${ }^{3}$, José- \\ Manuel Alija-Pérez ${ }^{3}$, María Teresa García-Ordás ${ }^{3}$ \\ ${ }^{1}$ SALBIS Research Group, Department of Electric, Systems and Automatics Engineering, Universidad de León, León, León, Spain \\ 2 Department of Electric, Systems and Automatics Engineering, Universidad de León, Leon, León, Spain \\ 3 SECOMUCI Research Group, Escuela de Ingenierías Industrial e Informática, Universidad de León, León, León, Spain \\ Corresponding Author: José Alberto Benítez-Andrades \\ Email address: jbena@unileon.es
}

With the growth that social networks have experienced in recent years, it is entirely impossible to moderate content manually. Thanks to the different existing techniques in natural language processing, it is possible to generate predictive models that automatically classify texts into different categories. However, a weakness has been detected concerning the language used to train such models. This work aimed to develop a predictive model based on BERT, capable of detecting racist and xenophobic messages in tweets written in Spanish. A comparison was made with different Deep Learning models. A total of five predictive models were developed, two based on BERT and 3 using other deep learning techniques, CNN, LSTM and a model combining CNN+LSTM techniques. After exhaustively analyzing the results obtained by the different models, it was found that the one that got the best metrics was BETO, a BERT-based model trained only with texts written in Spanish. The results of our study show that the BETO model achieves a precision of $85.22 \%$ compared to the $82.00 \%$ precision of the mBERT model. The rest of the models obtained between $79.34 \%$ and $80.48 \%$ precision. On this basis, it has been possible to justify the vital importance of developing native transfer learning models for solving Natural Language Processing (NLP) problems in Spanish. Our main contribution is the achievement of promising results in the field of racism and hates speech in Spanish by applying different deep learning techniques. 


\title{
Detecting racism and xenophobia using deep learning models on Twitter data: CNN, LSTM and BERT
}

4 José Alberto Benítez-Andrades ${ }^{1}$, Álvaro González-Jiménez ${ }^{2}$, Álvaro López-Brea ${ }^{2}$, Jose Aveleira-Mata ${ }^{3}$, José Manuel Alija-Pérez ${ }^{3}$, and María Teresa García-Ordás ${ }^{3}$

\author{
Corresponding author: \\ José Alberto Benítez-Andrades ${ }^{1}$ \\ Email address: jbena@unileon.es
}

${ }^{1}$ SALBIS Research Group, Department of Electric, Systems and Automatics Engineering, Universidad de León, Campus of Vegazana s/n, León, 24071, León, Spain

${ }^{2}$ Department of Electric, Systems and Automatics Engineering, Universidad de León, Campus of Vegazana s/n, León, 24071, León, Spain

${ }^{3}$ SECOMUCI Research Group, Escuela de Ingenierías Industrial e Informática, Universidad de León, Campus de Vegazana s/n, C.P. 24071 León, Spain

\begin{abstract}
With the growth that social networks have experienced in recent years, it is entirely impossible to moderate content manually. Thanks to the different existing techniques in natural language processing, it is possible to generate predictive models that automatically classify texts into different categories. However, a weakness has been detected concerning the language used to train such models. This work aimed to develop a predictive model based on BERT, capable of detecting racist and xenophobic messages in tweets written in Spanish. A comparison was made with different Deep Learning models. A total of five predictive models were developed, two based on BERT and 3 using other deep learning techniques, CNN, LSTM and a model combining CNN+LSTM techniques. After exhaustively analyzing the results obtained by the different models, it was found that the one that got the best metrics was BETO, a BERT-based model trained only with texts written in Spanish. The results of our study show that the BETO model achieves a precision of $85.22 \%$ compared to the $82.00 \%$ precision of the mBERT model. The rest of the models obtained between $79.34 \%$ and $80.48 \%$ precision. On this basis, it has been possible to justify the vital importance of developing native transfer learning models for solving Natural Language Processing (NLP) problems in Spanish. Our main contribution is the achievement of promising results in the field of racism and hates speech in Spanish by applying different deep learning techniques.
\end{abstract}

\section{INTRODUCTION}

In recent years, the use of social networks such as Twitter, Facebook or Instagram, as well as other community forums, is generating hateful conversations among users (Del Vigna et al., 2017; Watanabe et al., 2018; Zhang and Luo, 2019). The fact that people can comment anonymously is one of the factors that leads to the spread of hate speech (Barlett, 2015).

Within hate crimes, some countries are particularly interested in studying racist and xenophobic crimes (Sayan, 2019; Rodríguez Maeso, 2018). For example, in the UK, hate speech towards different Muslim and other immigrant communities has increased (Anonymous, 2017; Travis, 2017). A link was found between the rise of racism and the exit from the EU or the Manchester bombings. In other countries, such as Spain, the Congress of Deputies has recently approved the "Non-legislative motion on preventing the spread of hate speech in the digital space" (Congreso de los Diputados of Spanish Government, 2020). In short, the fight against racism and xenophobia are issues of international concern.

Some studies are currently working on the classification of texts for the detection of racism or xenophobia (Kumari et al., 2021; Al-Hassan and Al-Dossari, 2019; Alotaibi and Abul Hasanat, 2020; 
Plaza-Del-Arco et al., 2020; Chaudhry, 2015; Konstantinidis et al., 2017; Sazzed, 2021). To do this, they have used different techniques in the field of artificial intelligence and, more specifically, within natural language processing. One of the most widely used techniques for this purpose is text classification on different datasets obtained from some social networks, Twitter being one of the most widely used (Saha et al., 2020; Garcia and Berton, 2021).

In order to classify texts using machine learning techniques and, more specifically, deep learning, techniques are often used such as Convolutional Neural Network (CNN), Recurrent Neural Network (RNN) and Hierarchical Attention Network (HAN). Within these techniques, Long Short-Term and, more specifically, Bi-directional Long Short-Term (BiLSTM) are the most responsive. Analysing the latest developments in this regard are the BERT-based approaches.

However, one of the barriers encountered in detecting racist and xenophobic tweets is language. There are deep learning models that detect racist or xenophobic texts in English. Nevertheless, so far, no pretrained model has been found with texts in Spanish and there are studies showing that classification models trained with native language texts give better results (Gutiérrez-Fandiño et al., 2021; Pomares-Quimbaya et al., 2021; Kamal et al., 2021; Sharma et al., 2022; Velankar et al., 2021). Therefore, the main objectives of this research are those shown below:

- Obtain a set of texts in Spanish related to racism and xenophobia. Preprocess the data set and label said texts in a binary category, giving a value of 1 if the message is xenophobic or racist, and 0 when it is not.

- Apply different deep learning models and determine which of them is the one with the highest accuracy and precision.

In this paper, we propose a dataset related to racism and xenophobia in the Spanish language and we propose different models based on deep learning techniques to detect racist or xenophobic texts.

The paper is organized as follows. Related work is presented in the section 2. The methodology of the different proposed techniques is detailed in section 3. In section 4 the setup of different deep learning techniques and the results, showing a comparative between the different techniques are explained. Finally, results have been discussed in section 5 and we conclude in section 6 .

\section{RELATED WORK}

Within the state of the art, two different branches can be analysed, the Spanish language datasets publicly available on the web and the natural language processing techniques most commonly used in text classification.

\subsection{Existing Datasets}

While it is possible to find a large number of datasets in English that are properly labelled for the detection of racism, and especially hate speech, it is extremely difficult to find datasets in Spanish with these same characteristics.

A total of two Spanish datasets related to hate speech has been found, and it is completely impossible to find one related solely and exclusively to racism/xenophobia. Nevertheless, both datasets have been explored: HaterNet (Pereira-Kohatsu et al., 2019) and HatEval (Basile et al., 2019).

Other datasets were found that also contained texts in Spanish, but were not exclusively in Spanish, as in the case of the PHARM project, where the dataset is composed of texts in Spanish, Italian and Greek (Vrysis et al., 2021).

\subsubsection{HaterNet}

The first of the two datasets has been obtained thanks to HaterNet (Pereira-Kohatsu et al., 2019), an intelligent system used by the National Office for the Fight against Hate Crimes, belonging to the Spanish Ministry of Interior (Ministerio Interior, 2019).

This dataset has a total of 6,000 labelled tweets indicating the presence or not of hate speech. Among the hate speech, there are, unsurprisingly, some tweets in which racism can be seen. However, they represent a rather low percentage of the total number of labelled tweets, which in practice makes the dataset invalid for training the model proposed in this project. 
Not only are there tweets on topics that differ completely from racism, but the labelling criteria are also somewhat confusing. For these reasons, this dataset has been completely discarded for use in the training and subsequent validation of the predictive model.

\subsubsection{HatEval}

The second dataset has been obtained thanks to HatEval (Basile et al., 2019), a competition organised by SemEval (International Workshop on Semantic Evaluation), whose main objective was to develop a predictive model for the detection of anti-female and anti-immigration hate speech on Twitter. In order to access this dataset, it is necessary to request permission from one of its creators as shown in the repository https://github.com/msang/hateval.

The dataset consists of two different sets of tweets. The first one contains tweets written in English, which makes it completely unusable for the purpose of this project. The second one, in turn, contains 5,000 tweets written in Spanish in the training set and 1,600 in the test set.

Of these 5,000 tweets, a total of 1,971 are related to racism, which makes the set, at least initially, valid.

As regards the structure of the dataset, for each tuple, a text followed by three binary variables can be found. The first, called HS, denotes whether the tweet expresses hate speech or not. The second, known as TR, indicates whether the victim is a single person or a group of people. Finally, the variable called AG indicates whether the tweet is expressed in an aggressive way or not.

After looking closely at the dataset, it was determined that $987(50,07 \%)$ tweets out of 1,971 were mislabelled. There are tweets from some people denouncing racism that are labelled as racist. The same goes for some comments that are clearly ironic. On other occasions, tweets that have nothing to do with racism (in South America the word "sudaca" is used to refer to the subcontinent itself) or that are simply not racist are labelled as hate crimes.

Because of all these labelling failures, it has been determined that this dataset is also not valid for the purpose of this project.

Therefore, after having thoroughly analysed both datasets, it has been concluded that it is strictly necessary to build a suitable dataset. The whole process of building this dataset can be found in section 3 .

\subsection{Text classification}

The techniques that can be used to create models of automatic classification of a text are very varied. However, it is possible to group them into 3 main types of techniques: conventional machine learning, deep learning and transfer learning.

\subsubsection{Conventional Machine Learning}

Among the various conventional machine learning techniques used in text classification , and especially in the detection of hate speech on the Internet, support vector machines (SVM) and logistic regression stand out.

SVMs (Cortes and Vapnik, 1995) are extremely accurate and effective in classifying texts (Ahmad et al., 2018; Hasan et al., 2019). One of their main advantages is that, unlike most other techniques, they perform particularly well on small training sets. Commonly, when using this technique, text features are usually obtained by applying TF-IDF or word embeddings, or another technique with a similar functionality such as Part of speech (POS).

Logistic regression (Lakshmi et al., 2018; Br Ginting et al., 2019) is a type of regression analysis used to predict the outcome of a categorical variable given a set of independent variables. Like SVMs, it is also necessary to extract text features with one of the aforementiond techniques before training begins. Unlike SVMs, they perform significantly worse on small training sets.

\subsubsection{Deep learning}

Deep learning is a specific branch of machine learning whose main difference is that, unlike traditional machine learning algorithms, it is capable of continuing to learn as it receives more and more data, without stagnating.

Among the deep learning techniques most commonly used in text classification, convolutional neural networks (CNNs) and recurrent neural networks (RNNs) stand out.

Convolutional neural networks (Nedjah et al., 2019; Roy et al., 2020) are a type of neural network that, combined with supervised learning, process layers by mimicking the human eye, allowing them to differentiate different features in the received inputs. Although they were specifically designed for 
computer vision, they have been shown to perform excellently on textual classification problems as well. When extracting text features, a stage prior to learning the network, it is common to use word embeddings.

Recurrent neural networks (Paetzold et al., 2019) are a type of neural network in which the connections between nodes form a directed graph along a temporal sequence. Among the different variants of this type of network, the Long Short-Term Memory Network (LSTM) (Talita and Wiguna, 2019; Bisht et al., 2020; Zhao et al., 2020; Zhang et al., 2021), which were specifically designed to avoid the problem of long-term dependency. As with CNNs, a correct extraction of text features prior to the learning period of the network, which is carried out by means of word embeddings, is of vital importance.

\subsubsection{Transfer learning}

Transfer learning is a machine learning technique in which the knowledge gained from carrying out a certain task is stored and then applied to a related problem. It is especially used for building models where a small amount of data is available for training and evaluation.

Within NLP tasks, the first attempted use of transfer learning was the creation of embeddings from large datasets such as Wikipedia. Although this was a major breakthrough in NLP, especially when training on very small datasets, there were still problems in differentiating the context in which words are written.

To solve this problem, a number of context-based pre-trained models such as Embedding from Language Models (ELMO) (Peters et al., 2018) and Bidirectional Encoder Representations from Transformers (BERT) (Devlin et al., 2019) have emerged, and a model very similar to the latter will be used in this project.

\subsection{Detecting racism and hate speeches in Spanish}

As regards the detection of racism, to date there is no model in Spanish developed for this specific purpose. However, research has been carried out on hate speech in Spanish.

The most important study on hate speech in Spanish is (Plaza-Del-Arco et al., 2020). It compares the performance of various NLP models in classifying tweets in the HatEval dataset. These models include SVM, linear regression (LR), Naive Bayes (NB), decision trees (DT), LSTM and a lexicon-based classifier. Of these, the best performing was an Ensemble Voting Classifier, which combines the output of several classifiers when making a prediction.

The second study (del Arco et al., 2021), by the same authors, compares the performance of other NLP models when classifying tweets from the HaterNet and HatEval datasets. These models include deep learning models such as LSTM, Bidirectional Long Short-Term Memory Networks (Bi-LSTM) and CNN, as well as transfer learning models such as mBert, XLM and BETO (Peters et al., 2018), all of which are based on BERT. The best performer on almost all metrics was BETO.

Finally, the study (Pereira-Kohatsu et al., 2019) presents HaterNet, an intelligent system that identifies and monitors the evolution of hate speech on Twitter. In this study, in addition to building the aforementioned HaterNet dataset, a series of comparisons are also made between different text classification models. These models include LDA, QDA, Random Forest, Ridge Logistic Regression, SVM and an LSTM combined with an MLP, the latter being the best performing.

After having analysed the different existing models, and having verified that there are no models specifically developed to detect racism in texts written in Spanish, and that the hate speech detection models have all been trained on the same two datasets, which have numerous mislabelled tweets, the development of new models for this purpose is more than justified.

\subsection{Summary of the literature review}

A summary of the literature review is shown in the table 1, specifying the important results found in each study analysed, as well as the weaknesses found in relation to the objectives presented in our study. 
Table 1. Summary of the objectives and weaknesses of the literature review conducted.

\begin{tabular}{|c|c|c|}
\hline Reference & Findings & Weaknesses \\
\hline $\begin{array}{l}\text { (Ahmad et al., 2018; Hasan } \\
\text { et al., 2019; Lakshmi et al., } \\
\text { 2018; Br Ginting et al., 2019) }\end{array}$ & $\begin{array}{l}\text { Studies that corroborate good results in the task } \\
\text { of text classification using conventional machine } \\
\text { learning techniques (SVMs, Logistic regression). }\end{array}$ & $\begin{array}{l}\text { These articles are not focused on obtaining predic- } \\
\text { tive models for classifying categories of racism and } \\
\text { xenophobia in Spanish texts. }\end{array}$ \\
\hline (Pereira-Kohatsu et al., 2019) & $\begin{array}{l}\text { HaterNet: dataset of } 6,000 \text { labelled tweets as hate } \\
\text { speech or not. LDA, QDA, Random Forest, Ridge } \\
\text { Logistic Regression, SVM and an LSTM combined } \\
\text { with an MLP applied to HaterNet. }\end{array}$ & $\begin{array}{l}\text { Tweets labelled as racist, but not racist. Very small } \\
\text { percentage of tweets are related to racism, which is } \\
\text { one of the topics that interests us for this work. }\end{array}$ \\
\hline (Basile et al., 2019) & $\begin{array}{l}\text { HateEval: two datasets, one in english and the sec- } \\
\text { ond one in Spanish. Spanish dataset has 5,000 la- } \\
\text { belled tweets in the training set. }\end{array}$ & $\begin{array}{l}\text { Only } 1,971 \text { tweets are related to racism and } 987 \text { of } \\
\text { this subset were mislabelled. Tweets from some } \\
\text { people dnouncing racism that are labelled as racist. }\end{array}$ \\
\hline $\begin{array}{l}\text { (Nedjah et al., 2019; Roy } \\
\text { et al., 2020) }\end{array}$ & $\begin{array}{l}\text { Convolutional neural networks applied to different } \\
\text { problems. }\end{array}$ & $\begin{array}{l}\text { CNN applied to different problems, not only for } \\
\text { text classification and not focused on racism and } \\
\text { xenophobia. }\end{array}$ \\
\hline (Paetzold et al., 2019) & Theory about recurrent neural networks (RNN). & $\begin{array}{l}\text { Usually, training the BERT model from scratch on } \\
\text { similar dataset could produce much better result } \\
\text { (Sany et al., 2022; Shahri et al., 2020). }\end{array}$ \\
\hline $\begin{array}{l}\text { (Talita and Wiguna, 2019; } \\
\text { Bisht et al., 2020; Zhao et al., } \\
\text { 2020; Zhang et al., 2021) }\end{array}$ & $\begin{array}{l}\text { Long Short-Term Memory Network (LSTM) ap- } \\
\text { plied to different problems. }\end{array}$ & $\begin{array}{l}\text { Not all of them are focused on text classification. } \\
\text { Those that are, do not make comparisons with } \\
\text { BERT. }\end{array}$ \\
\hline (Plaza-Del-Arco et al., 2020) & $\begin{array}{l}\text { SVM, linear regression (LR), Naive Bayes (NB), } \\
\text { decision trees (DT), LSTM and a lexicon-based } \\
\text { classifier applied to a dataset composed by tweets } \\
\text { related to xenophobia and misogyny. }\end{array}$ & $\begin{array}{l}\text { The tweets are labelled with value } 1 \text { if speaks about } \\
\text { xenophobia or misogyny. This may bias the results } \\
\text { of the model. The best model was } 74.2 \% \text { of F1- } \\
\text { score. }\end{array}$ \\
\hline (del Arco et al., 2021) & $\begin{array}{l}\text { LSTM, Bidirectional Long Short-Term Memory } \\
\text { Networks (Bi-LSTM) and CNN, mBert, XLM and } \\
\text { BETO applied to HatEval and HaterNet. }\end{array}$ & $\begin{array}{l}\text { The dataset are biased because the subsets were } \\
\text { mislabelled. }\end{array}$ \\
\hline
\end{tabular}

\section{METHODOLOGY}

This section explains the methodology used in this research. In figure 1 it is possible to see the complete workflow of the work carried out.

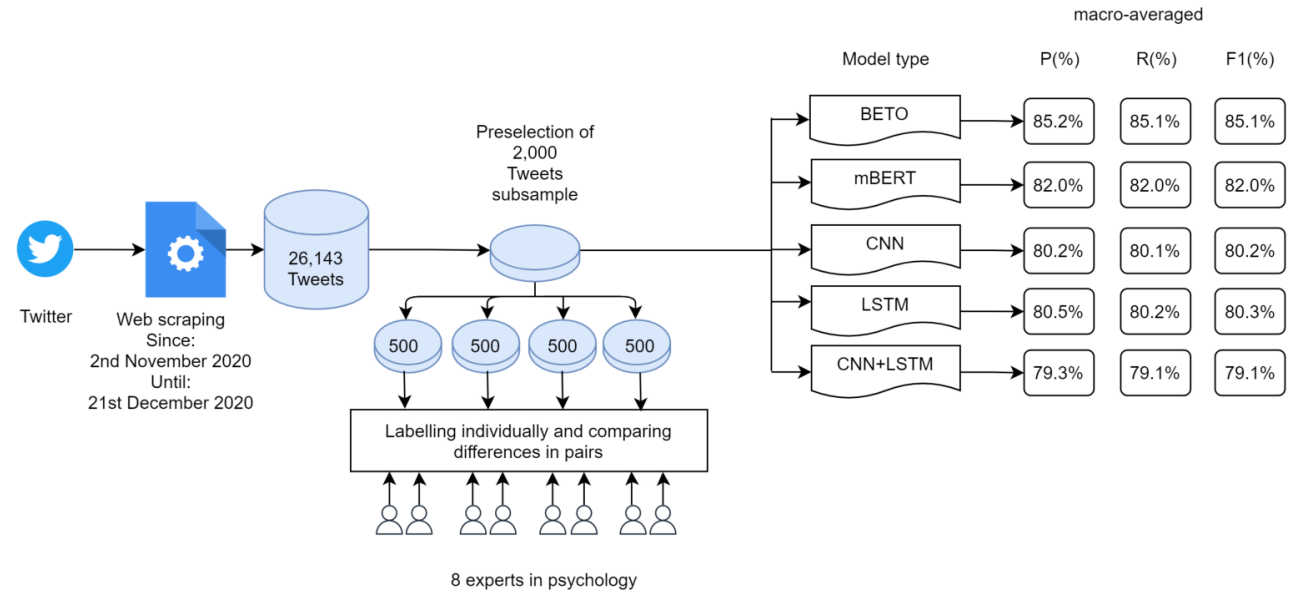

Figure 1. Workflow of the research conducted.

All the data used in the experiments, as well as the models generated and the source code are available in a Github repository at this link: https://doi.org/10.5281/zenodo.5188098 


\subsection{Dataset}

Having found that neither of the two public datasets in Spanish labelling the presence or absence of hate speech was sufficiently valid for training the proposed model, a proprietary dataset was constructed that could be fully adapted to the problem posed.

The Tweepy library (Roesslein, 2020) has been used to build this dataset. This library makes use of the Twitter API. The search patterns used for the construction of the dataset.

A keyword search was performed. These words are shown in table 2, as well as their English translation:

Table 2. Spanish keywords used to collect dataset and their translation to English language.

\begin{tabular}{c|c}
\hline Spanish Keyword & Translation to English \\
\hline moro, mora & moor \\
gitano, gitana, gypsy & gypsy \\
puto simio & fucking ape \\
fucking nigga \\
negro de mierda, negra de mierda, puto negro, puta negra \\
inmigración, inmigrante \\
patera & immigration, immigrant \\
small boat
\end{tabular}

A dataset of 26,143 tweets dating between 2nd November 2020 and 21st December 2020 was generated. Although for training purposes it is only necessary to have the text of the tweet and its classification, in order to carry out an analysis of the data, it was decided to store additional information such as Location, Number of followers, Number of followings, Total number of tweets, Date of user creation, Date of tweet creation, Number of retweets and Hashtags.

As far as data privacy is concerned, it is demonstrated that Cambridge Analytica is still alive and we can export people's behavioural characteristics without their consent just by acquiring publicly available data (Pitropakis et al., 2020; Kandias et al., 2013; Isaak and Hanna, 2018). This information, being public and anonymized, is exempt from the request for approval by an ethics committee (Eysenbach and Till, 2001).

\subsection{Preprocessing and labelling data}

After analysing the different articles in which predictive models are generated, it was decided to make use of a subset of data consisting of 2,000 tweets to be labelled in the two categories to be classified: racist tweets and non-racist tweets. The subset of data chosen was balanced, with 52\% of tweets labelled as non-racist and $48 \%$ of tweets labelled as racist.

From the initial set of 26,143 tweets, a pre-selection of 2,000 tweets was made by 4 students of computer engineering interpreting 50\% of them as belonging to each category: racist or xenophobic tweets and tweets that were neither racist nor xenophobic. Subsequently, labelling was carried out manually by eight experts in the field of psychology. The set of 2,000 tweets was divided into 4 subsets of 500 tweets that were labelled by 2 people individually. The pairs of experts then compared their labels and decided which label to assign to tweets where there might be some doubt as to whether or not they belonged to a category. Subsequently, a clustering phase of all labelled tweets was carried out.

Before generating this subset, a pre-processing of the data was carried out, which included the following tasks:

- Removal of extremely short tweets in which it is totally impossible to identify the presence or absence of racism.

- Removal of ironic tweets.

- Removal of tweets that are excessively badly written, making them difficult to understand or simply using invalid characters repeatedly.

- Conversion of all text to lowercase.

- Removal of URLs. 
- Elimination of unnecessary spaces.

- Elimination of user names.

- Elimination of unnecessary characters.

- Elimination of accents.

- Elimination of stopwords.

The following criteria were also taken into account when selecting the subset:

- Variety of terms in the dataset. While the filters used to capture the tweets are extremely varied, in practice some terms are much more popular than others. For example, the terms "inmigración" (immigration) or patera (small boat) appear in a much higher number of tweets than the term "negra de mierda" (fucking nigga). Therefore, if this factor is not taken into account when tagging tweets, we end up with a data set that is too poor with data that is too similar.

- Variety of meanings within the same term. Although many of the terms do not leave any doubt about their meaning, some of them may have different meanings which, if they are not both included, the behaviour of the model in the future when receiving input from these terms would be considerably reduced. An example of words to which this criterion applies directly is: "mora" (moor), "mena" (unaccompanied foreign minor) or "panchito" (spic).

\subsection{BERT Models}

BERT (Devlin et al., 2019) makes use of Transformers (Vaswani et al., 2017), a deep learning model proposed by researchers at Google and the University of Toronto in 2017 that has particular application in the field of natural language processing.

Like Recurrent Neural Networks (RNN), Transformers is designed to work with sequential data. In a similar way to humans, it is capable of processing natural language, serving to carry out tasks as diverse as translation or text classification. However, unlike RNNs, Transformers do not require sequential data, text in this case, to be processed in order.

This means that, when receiving a text as input, it is not necessary to process the beginning of the text before the end, which allows for much greater parallelisation and, therefore, reduces training times considerably.

Transformers have been designed using the concept of the attention mechanism, which itself was designed to memorise long sentences in machine translation tasks.

At the architectural level, it is based on an encoder-decoder architecture in which the encoders consist of a set of encoding layers that iteratively process the input layer by layer. In turn, the decoders consist of a set of decoding layers that do the same at the output of the encoder.

Thus, when Transformers receive a text, it passes through a stack of encoders. The output obtained at the last encoder is passed to each of the decoders that make up the stack of decoders, resulting in a final output. A very high-level representation of Transformers can be found in the figure 2 .

Each encoder consists of two main components, an attention mechanism called self-attention and a feed forward neural network.

The encoder receives a list of numeric vectors as input that flows through the self-attention layer. This layer helps the encoder to take into account the rest of the words in the sentence before encoding each word. Although the example in the figure above is very simple, the encoder, before encoding the word "Thinking", would take into account that this word is accompanied by the word "Machines" when generating the z1 vector, just as it would do the same with the word "Machines" when generating the $\mathrm{z} 2$ vector.

The output obtained for each of the input vectors, a list of $\mathrm{z}$ vectors in this case, is passed through a pre-fed neural network, which will generate an output for each $\mathrm{z}$ input vector. This output, which in the figure is represented as $r$, becomes the input to the next encoder, which will perform the same process as described above.

Finally, it should be noted that each encoder has a series of residual connections that prevents the output of one layer from being processed by the next layer. These mechanisms are particularly relevant in 


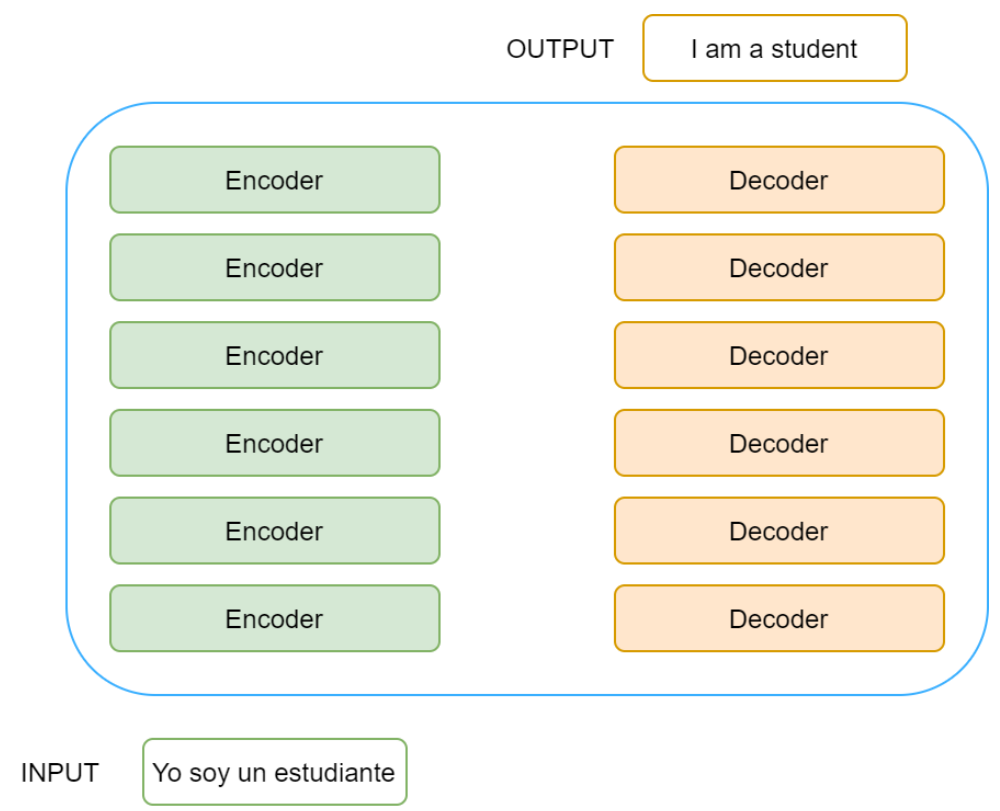

Figure 2. High-level representation of Transformers.

neural networks that have many hidden layers, allowing certain layers to be left unused if necessary. In addition, it should be noted that a normalisation process is applied to the output of each layer.

Since its publication, it has become the best existing solution for a large number of NLP problems. The most special feature of the model is the way in which it deals with the meaning of words depending on the context in which they are used, using an architecture based on Transformers, which will be explained in due course.

BERT's first differentiating factor is the difference from older embedding generation techniques such as Word2Vec, Glove or FastText.

The embeddings generated using the aforementioned techniques are context-independent, i.e. each word has a unique vector that defines it whatever the context in which it is used. This means that all the different meanings of a given word are combined into a single vector.

\subsection{Metrics to evaluate results}

To evaluate the results obtained in each of the models developed, 3 different metrics have been used: Precision (P), Recall (R) and F1-score (F1).

\subsubsection{Precision}

Precision is a metric used to calculate what percentage of the positive samples (which in this particular case equals the samples labelled as racist) have been properly classified. The formula used to calculate this metric is as follows, where $\mathrm{c}$ is equal to the class $(0=$ Non-racist, $1=$ Racist $), \mathrm{TP}=$ True positive, FP $=$ False positive and $\mathrm{FN}=$ False negative:

$$
P(c)=\frac{T P}{T P+F P}
$$

\subsubsection{Recall}

Recall, in turn, is a metric used to calculate what percentage of the samples classified as positive have been properly classified. The formula used to calculate this metric is as follows, where $\mathrm{c}$ is equal to the class $(0=$ Non-racist, $1=$ Racist $), \mathrm{TP}=$ True positive and FN $=$ False negative:

$$
R(c)=\frac{T P}{T P+F N}
$$




\subsubsection{F1-score}

F1-score is a metric used to calculate the effectiveness of a classifier by taking into account its accuracy and recall values. F1 assumes that the two metrics used are of equal importance in calculating effectiveness. If one of them is more important than the other, a different formula $F_{\beta}$ would have to be used. The formula used to calculate this metric is as follows, where $\mathrm{P}$ equals the precision value and $\mathrm{R}$ equals the recall value:

$$
F_{1}=\frac{2 * P * R}{P+R}
$$

\section{EXPERIMENTS AND RESULTS}

\subsection{Experimental Setup}

As part of the development of this project, a total of 5 different predictive models have been developed. Two are transfer learning models and three are deep learning models.

Within the transfer learning models, a model based on BETO has been developed, which is very similar to BERT trained in Spanish, and a model based on mBERT, a BERT checkpoint that can be used on texts in a large number of different languages. With respect to deep learning models, a model has been developed that implements a convolutional neural network, another model that implements a recurrent neural network (LSTM) and a last model that fuses a convolutional neural network with another recurrent neural network.

\subsubsection{BETO model}

BETO (Cañete et al., 2020) is a transfer learning model that has been trained in the same way as BERT, except that it has been trained using texts written in Spanish instead of English, including Wikipedia entries, subtitles of series and movies, and even news. The specific technique with which it has been trained is called Whole Word Masking, a technique very similar to the Masked Language Model in which instead of hiding random tokens, it ensures that the hidden tokens always constitute a word, which means that if a token corresponding to a sub-word is hidden, the rest of the tokens that make up the whole word are also hidden. If we make a comparison between BETO and the different BERT models, we could say that it is a model extremely similar to the base BERT, given that it has 12 layers of encoders in its architecture.

Within BETO, there are two different models, one that has been trained using words containing both upper and lower case letters (original texts therefore) and another one that has been trained using only words written in lower case letters (texts are processed to comply with this premise). Although both models offer great results, depending on the problem to be solved, it is more appropriate to use one rather than the other. In this particular case, given that the dataset used is made up of tweets (where people are not at all careful about the way they express themselves), the uncased model is the one that performs better. After carrying out a large number of tests with both models, it has been found that the results obtained by this model are between $0.5 \%$ and $1 \%$ better, which justifies the selection of this model.

\subsection{2 mBERT model}

mBERT (Multilingual BERT) (Devlin et al., 2019) is a transfer learning model that has been trained on Wikipedia texts written in 104 different languages, including Spanish. Like BETO, it also has an identical architecture to the BERT base, with 12 layers of encoders, and two different models, one trained with texts containing both lowercase and uppercase letters and the other trained with texts containing only words written in lowercase letters. Contrary to BETO, after several tests, it has been found that the cased model performs slightly better than the uncased model. However, due to some problems in the tokenisation process, it has been necessary to convert all text to lower case even though the selected model is case-sensitive.

Multilingual models frequently encounter the common problem of language detection. This type of model does not usually have any mechanism or system that allows the detection of the language in which the texts that make up the input are written, which means that on many occasions the tokenizer makes mistakes when dividing the texts into tokens. If we add to this the fact that this type of model does not have mechanisms that allow words from different languages with the same meaning to be represented in a similar way in the vector space, everything seems to indicate that the results obtained after training these 
models are worse than those obtained by native models, that is, those designed to work with texts written in a single language (as is the case of BETO in Spanish or BERT in English).

\subsubsection{CNN model}

This model is made up of the following layers:

- Embedding layer.

- 3 convolutional layers each followed by a MaxPooling layer. 256, 128 and 64 filters. MaxPooling of $2 \times 2$.

- Final output layer with a single neuron, in charge of classifying the sample.

The schematic architecture of the model is shown in figure 3.

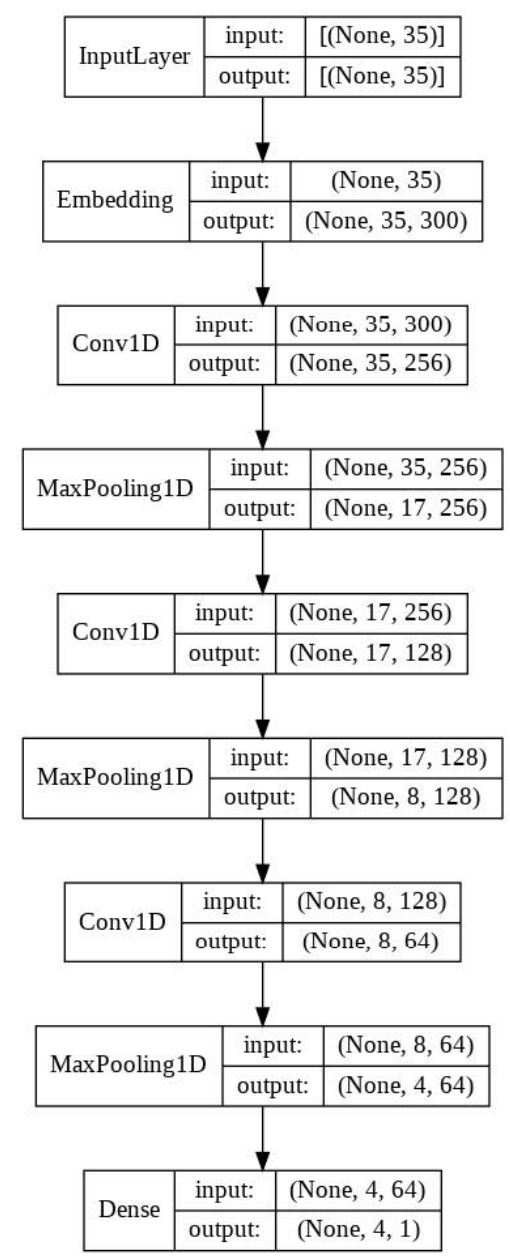

Figure 3. $\mathrm{CNN}$ architecture scheme 


\subsubsection{LSTM model}

This model consists of the following layers:

- Embedding layer.

- LSTM layer with 64 units.

- 2 fully connected layers with dropout between them.

- Final output layer with a single neuron, in charge of classifying the sample.

The schematic architecture of the model is shown in figure 4 .

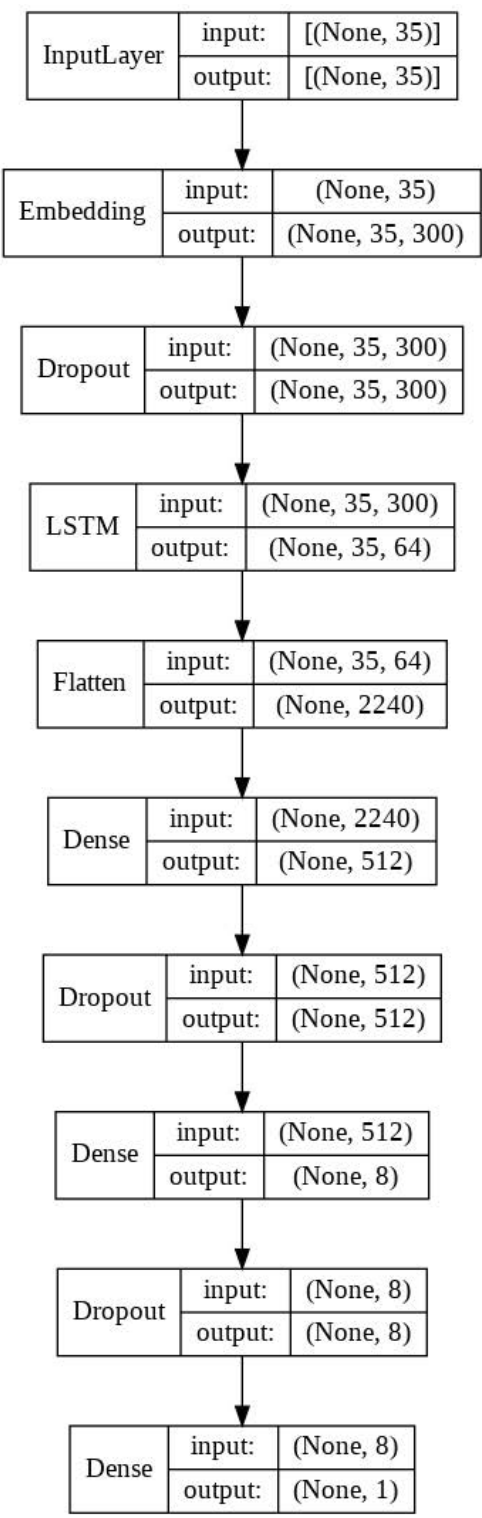

Figure 4. LSTM architecture scheme 


\subsubsection{CNN + LSTM model}

This model consists of the following layers:

- Embedding layer.

- Convolutional layer with 64 filters.

- LSTM layer with 128 filters.

- Final output layer with a single neuron, in charge of classifying the sample.

The schematic architecture of the model is shown in figure 5 .

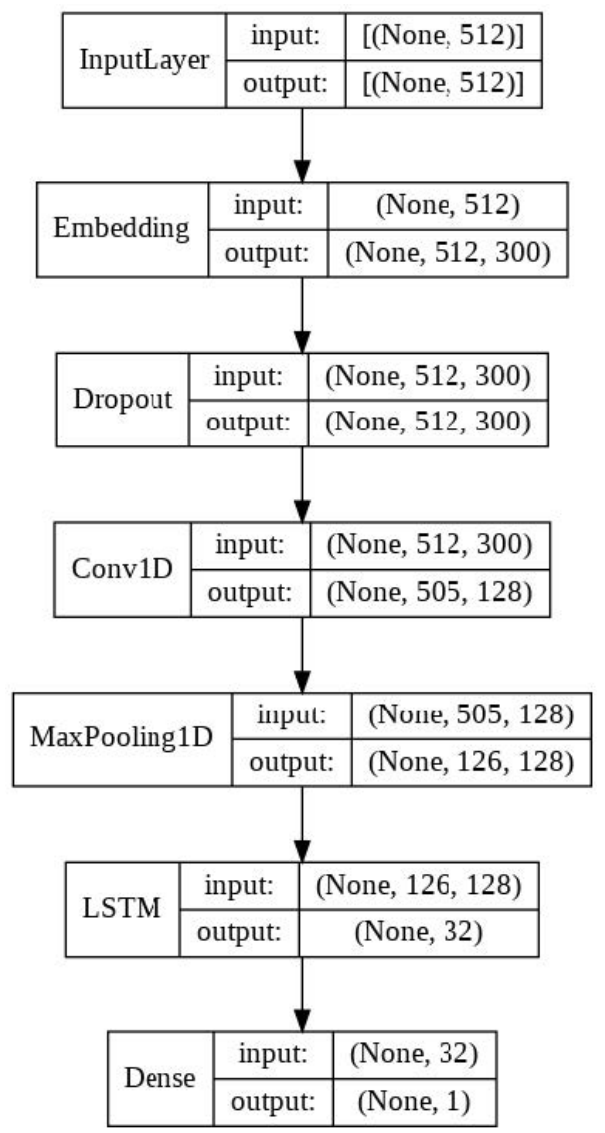

Figure 5. CNN + LSTM architecture scheme 


\subsection{Transfer Learning Parameter Optimisation (BETO and mBERT)}

In order to ensure that the results obtained by the transfer learning models are as high as possible, a series of tests have been carried out in which the performance of both models has been tested as a function of the value taken by the various hyperparameters.

Although the number of hyperparameters that can be customised is really high, in practice it is totally impossible to try to modify all of them given the exponential growth experienced in the number of combinations. Therefore, it has been necessary to select those parameters that have a greater relevance in the behaviour of the model (Sun et al., 2019).

The parameters selected were as follows:

- Type of model: Cased or uncased.

- Number of epochs: 2, 4 and 8 . The number of epochs dictates the number of times the model will process the entire training set.

- Batch size: 8, 16, 32 and 64. This parameter indicates the number of samples to be processed by the model until an internal update of the model weights is performed.

- Optimiser: Adam and Adafactor. Mechanisms used to manage the update of the model weights.

- Learning rate: $0.00002,0.00003$ and 0.00004. Parameter that determines the step size when performing an update in the model with a view to approaching a minimum in a loss function.

As the dataset is made up of tweets, the length of which is generally limited to between 3 and 35 words, the maximum sequence size is not so relevant. However, if we were working with other types of text, it would be another parameter to be modified.

The different possible combinations tested (a total of 144) and the best parameters found for each of the two models can be found in the table 3 .

Table 3. The best transfer learning hyperparameters

\begin{tabular}{cccc}
\hline Hyperparameter & Options & BETO & mBERT \\
\hline Model type & [cased, uncased] & uncased & cased \\
Epochs & {$[2,4,8]$} & 8 & 4 \\
Batch size & {$[8,16,32,64]$} & 8 & 8 \\
Optimizer & [Adam, Adafactor] & Adam & Adam \\
Learning rate & {$[2 \mathrm{e}-5,3 \mathrm{e}-5,4 \mathrm{e}-5]$} & $4 \mathrm{e}-5$ & $4 \mathrm{e}-5$ \\
\hline
\end{tabular}

The most optimal configurations for both models are practically identical, with the exception of the model type and number of epochs. While in the case of BETO it is the uncased model that obtains the best results, in mBERT it is the cased model that obtains the best results, although the difference is minimal. In turn, in relation to the number of epochs, BETO obtains better results with 8 and mBERT with 4 .

\subsection{Optimisation of deep learning parameters (CNN, LSTM and CNN + LSTM)}

In order to ensure that the results obtained by the deep learning models are as high as possible, a series of tests have been carried out in which the performance of the models has been tested depending on the value of certain strategically selected parameters.

As in BERT, the number of parameters to be modified is really high, much higher still in this case since some factors such as the number of hidden neurons in each layer greatly influence the performance of the models. Although several tests have been carried out on both the structure of the different models and the number of hidden neurons in each layer, it is more important to focus on the remaining parameters that have been modified.

The selected parameters have been the following:

- Batch size: 16, 32, 64, 128.

- Dropout: 0.25, 0.5.

- Optimizador: Adam, SGD. 
- Función de activación: Relu, Tanh.

- Tasa de aprendizaje: $0.01,0.02,0.001,0.002$.

On numerous occasions, the number of epochs is also a parameter to be modified. However, in all the models pertaining to this project, we have chosen to use a mechanism called EarlyStopping, which is a regularisation mechanism used to avoid overfitting and which consists of stopping training when the control metrics of the validation set begin to decay.

The different possible combinations tested (a total of 64 in the CNN model and 128 in the LSTM and CNN+LSTM models) and the best parameters for each of the three models can be found in table 4 .

Table 4. The best deep learning hyperparameters

\begin{tabular}{ccccc}
\hline Hyperparameter & Options & CNN & LSTM & CNN+LSTM \\
\hline Batch size & {$[16,32,64,128]$} & 32 & 64 & 64 \\
Dropout & {$[0.25,0.5]$} & N/A & 0.5 & 0.5 \\
Optimizer & [Adam, SGD] & Adam & Adam & Adam \\
Activation function & {$[$ Relu, Tanh] } & Relu & Relu & Relu \\
Learning rate & {$[1 \mathrm{e}-2,2 \mathrm{e}-2,1 \mathrm{e}-3,2 \mathrm{e}-3]$} & $2 \mathrm{e}-3$ & $2 \mathrm{e}-3$ & $2 \mathrm{e}-3$ \\
\hline
\end{tabular}

The most optimal configurations for the three models are very similar, sharing values in optimiser, activation function and learning rate. Dropout, which is not present in the proposed CNN architecture, is identical in the other two models.

\subsection{Hardware and Software used for the experiments}

To perform the pre-processing of the tweets and apply the deep learning techniques, a Jupyter notebook, Python 3.6 was used and run on a computer with the following characteristics: Intel(R) Core(TM) i7-9700K CPU @ 3.60GHZ, 32.0GB RAM and an NVIDIA GeForce RTX 2080 6GB graphics card.

The spaCy and nltk libraries were used to pre-process the text content.

\subsection{Results}

The following table 5 shows the results obtained after numerous runs of the various predictive models. The runtime column refers to the time spent training and validating the model.

Table 5. Results obtained for all models

\begin{tabular}{c|ccc|ccc|ccc|c}
\hline Model & \multicolumn{3}{|c|}{ Non-racist } & \multicolumn{3}{c|}{ Racist } & \multicolumn{3}{c|}{ macro-averaged } & runtime \\
\hline & P(\%) & $\mathbf{R}(\%)$ & $\mathbf{F 1}(\boldsymbol{\%})$ & $\mathbf{P}(\boldsymbol{\%})$ & $\mathbf{R}(\boldsymbol{\%})$ & $\mathbf{F 1}(\boldsymbol{\%})$ & $\mathbf{P}(\boldsymbol{\%})$ & $\mathbf{R}(\boldsymbol{\%})$ & $\mathbf{F 1}(\boldsymbol{\%})$ & \\
\hline BETO & $\mathbf{8 4 . 2 8}$ & $\mathbf{8 7 . 3 0}$ & $\mathbf{8 5 . 7 6}$ & $\mathbf{8 6 . 1 7}$ & $\mathbf{8 2 . 9 4}$ & $\mathbf{8 4 . 5 2}$ & $\mathbf{8 5 . 2 2}$ & $\mathbf{8 5 . 1 2}$ & $\mathbf{8 5 . 1 4}$ & $1,230 \mathrm{~s}$ \\
mBERT & 83.28 & 81.11 & 82.18 & 80.73 & $\mathbf{8 2 . 9 4}$ & 81.82 & 82.00 & 82.02 & 82.00 & $1,129 \mathrm{~s}$ \\
CNN & 80.13 & 81.43 & 80.78 & 80.21 & 78.84 & 79.52 & 80.17 & 80.14 & 80.15 & $840 \mathrm{~s}$ \\
LSTM & 78.90 & 84.04 & 81.39 & 82.05 & 76.45 & 79.15 & 80.48 & 80.24 & 80.27 & $844 \mathrm{~s}$ \\
CNN+LSTM & 77.58 & 83.39 & 80.38 & 81.11 & 74.74 & 77.80 & 79.34 & 79.07 & 79.09 & $938 \mathrm{~s}$ \\
\hline
\end{tabular}

BETO offers the best results in each and every one of the metrics calculated, obtaining a wide advantage over the rest of the models. The confusion matrices of the two best-performing models are shown in figure 6.

As regards to the most important metric of all, Macro F1-score, in which all classes have the same importance in determining the effectiveness of the model, BETO obtains $85.14 \%$, improving by more than 3 points over the other transfer learning model developed (mBERT) and between 5 and 6 points over the deep learning models. Several conclusions can be drawn from these results. 

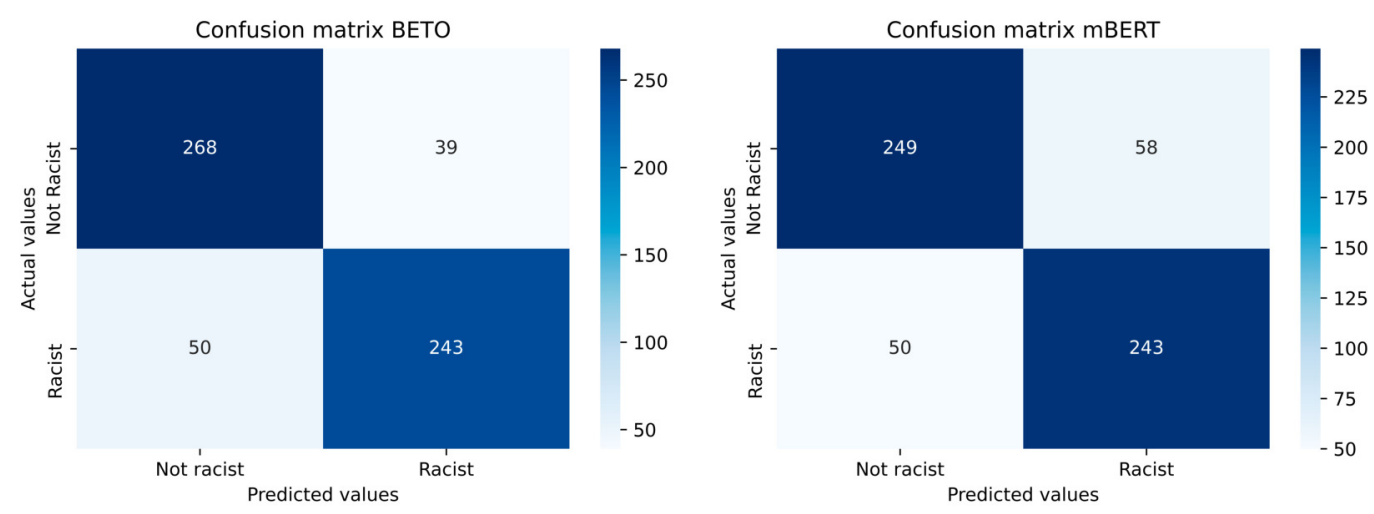

Figure 6. Confusion matrices of the best performing models (BETO and mBERT).

\section{CRITICAL EVALUATION AND COMPARISON OF THE RESULTS}

Firstly, comparing the two transfer learning models, it can be seen that the native model (BETO) performs substantially better than the multilingual model (mBERT). The main reasons for this difference between the two models are as follows:

- Vocabulary difference between the two models: As BETO was trained with texts written in Spanish and mBERT with texts written in 104 different languages, the number of Spanish words in the two models differs greatly, which means that the percentage of words in the dataset present in the models' vocabulary of the models is also very different. As these are transfer learning models, where a generic model is used to solve a specific problem, the difference in the word coverage of the dataset has a significant impact on the models' results.

- Difference in the tokenisers: While BETO has a tokeniser that is solely and exclusively responsible for tokenising texts written in Spanish, mBERT has a generic tokeniser that does not even have a mechanism for detecting the language in which the text it is processing is written. This means that on numerous occasions tokenisation in mBERT is carried out erroneously by recognising Spanish words as words that exist in other languages.

With respect to the deep learning models, although they perform considerably worse than the transfer learning models (especially BETO), it should be noted that the results obtained are really good if we take into account the simplicity of the models developed and the small amount of data in the training set (only 1,400 samples).

Among the factors that influence the poorer performance of these models are the following:

- Single embeddings for several meanings of the same word: Although pre-trained embeddings are used which have a coverage of $82.4 \%$ of the words (an exceptionally good figure), all meanings of the same word are represented by the same numeric vector. This means that, for words in this situation, their numeric vector is influenced by all the different meanings of the word, which causes the accuracy of the model to be reduced slightly.

- Context independence: Unlike transfer learning models, deep learning models do not have such an effective mechanism for representing words based on their context (RNNs have one, but it is nowhere near as effective as that implemented by BERT-based systems). This makes it often a bit complex for such models to take into account a word into account its specific context, which in addition to the unique embeddings for each word leads to poorer model results.

Taking into account all of the above, it is worth highlighting that BETO is, without a doubt, the model that best solves the problem posed in this research, also demonstrating how important it is to develop native transfer learning models, as they obtain better results than multilingual models.

In our research, BETO was the best performing model. Deep learning models generally operate as black boxes, so in some cases it is difficult to reason why some perform better or worse. However, our 
performance results are consistent with those obtained in other studies (del Arco et al., 2021; GutiérrezFandiño et al., 2021; Pomares-Quimbaya et al., 2021) in which BETO was also the best performing model. Although BETO and mBERT have very similar architectures, BETO was trained on Spanish data and mBERT was pre-trained on 104 languages. In this case it is evident, as in other articles that also make use of them (Gutiérrez-Fandiño et al., 2021; Pomares-Quimbaya et al., 2021) that for the present problem, the model trained specifically with the same language as the dataset for which an efficient solution is to be found offers better results.

\section{CONCLUSIONS}

In this research, the two objectives initially set have been completed:

- Messages on the Twitter platform containing words related to racism were obtained. A subsample of 2,000 messages was labelled, resulting in a balanced dataset.

- Different predictive models were generated using NLP techniques. These models were based on deep learning (CNN, LSTM and CNN+LSTM) and on transfer learning models (BERT). The best performing model was based on BERT, namely BETO with a precision of $85.22 \%$.

This fact justifies the need for the development of native transfer learning models. Having been trained with texts written in a single language instead of dozens of languages, the vocabulary of native models is far superior to that of multilingual models, which translates into greater effectiveness in the vast majority of situations.

This research shows preliminary results that need to be further investigated by improving some weaknesses, e.g. the size of the dataset used. Future research will increase the dataset, to achieve a more robust validation of the model presented in this article. Following more robust validation, it is intended to add new languages and to integrate these models into web applications that can be useful to society. Other limitations include, as in other studies that present text classification models, the need to retrain the model to include new terms generated by society over time. In addition to this, it is proposed to implement the model in an application that helps to automatically detect racism in different websites and to present results and validation of the complete system.

\section{REFERENCES}

Ahmad, M., Aftab, S., Bashir, M. S., and Hameed, N. (2018). Sentiment analysis using svm: A systematic literature review. International Journal of Advanced Computer Science and Applications, 9(2).

Al-Hassan, A. and Al-Dossari, H. (2019). Detection of Hate Speech in Social Networks: A Survey on Multilingual Corpus. In Computer Science \& Information Technology(CS \& IT), pages 83-100. AIRCC Publishing Corporation.

Alotaibi, A. and Abul Hasanat, M. H. (2020). Racism Detection in Twitter Using Deep Learning and Text Mining Techniques for the Arabic Language. Proceedings - 2020 1st International Conference of Smart Systems and Emerging Technologies, SMART-TECH 2020, pages 161-164.

Anonymous (2017). Finsbury park attack: son of hire boss held over facebook post. BBC News.

Barlett, C. P. (2015). Anonymously hurting others online: The effect of anonymity on cyberbullying frequency. Psychology of Popular Media Culture, 4(2):70-79.

Basile, V., Bosco, C., Fersini, E., Nozza, D., Patti, V., Rangel Pardo, F. M., Rosso, P., and Sanguinetti, M. (2019). SemEval-2019 task 5: Multilingual detection of hate speech against immigrants and women in Twitter. In Proceedings of the 13th International Workshop on Semantic Evaluation, pages 54-63, Minneapolis, Minnesota, USA. Association for Computational Linguistics.

Bisht, A., Singh, A., Bhadauria, H. S., Virmani, J., and Kriti (2020). Detection of Hate Speech and Offensive Language in Twitter Data Using LSTM Model BT - Recent Trends in Image and Signal Processing in Computer Vision, pages 243-264. Springer Singapore, Singapore.

Br Ginting, P. S., Irawan, B., and Setianingsih, C. (2019). Hate speech detection on twitter using multinomial logistic regression classification method. In 2019 IEEE International Conference on Internet of Things and Intelligence System (IoTaIS), pages 105-111.

Cañete, J., Chaperon, G., Fuentes, R., Ho, J.-H., Kang, H., and Pérez, J. (2020). Spanish pre-trained bert model and evaluation data. In PMLADC at ICLR 2020. 
Chaudhry, I. (2015). Hashtagging hate: Using Twitter to track racism online. First Monday, 20(2).

Congreso de los Diputados of Spanish Government, A. (2020). Proposición no de ley sobre la prevención de la propagación de discursos de odio en el espacio digital. [Online; posted 16-October-2020].

Cortes, C. and Vapnik, V. (1995). Support-Vector Networks. Machine Learning, 20(3):273-297.

del Arco, F. M. P., Molina-González, M. D., Ureña-López, L. A., and Martín-Valdivia, M. T. (2021). Comparing pre-trained language models for spanish hate speech detection. Expert Systems with Applications, 166:114120.

Del Vigna, F., Cimino, A., Dell'Orletta, F., Petrocchi, M., and Tesconi, M. (2017). Hate me, hate me not: Hate speech detection on Facebook. CEUR Workshop Proceedings, 1816:86-95.

Devlin, J., Chang, M.-W., Lee, K., and Toutanova, K. (2019). BERT: Pre-training of deep bidirectional transformers for language understanding. In Proceedings of the 2019 Conference of the North American Chapter of the Association for Computational Linguistics: Human Language Technologies, Volume 1 (Long and Short Papers), pages 4171-4186, Minneapolis, Minnesota. Association for Computational Linguistics.

Eysenbach, G. and Till, J. E. (2001). Ethical issues in qualitative research on internet communities. BMJ, 323(7321):1103-1105. Publisher: BMJ Publishing Group Ltd _eprint: https://www.bmj.com/content/323/7321/1103.full.pdf.

Garcia, K. and Berton, L. (2021). Topic detection and sentiment analysis in Twitter content related to COVID-19 from Brazil and the USA. Applied Soft Computing, 101:107057.

Gutiérrez-Fandiño, A., Armengol-Estapé, J., Pàmies, M., Llop-Palao, J., Silveira-Ocampo, J., Carrino, C. P., Gonzalez-Agirre, A., Armentano-Oller, C., Rodriguez-Penagos, C., and Villegas, M. (2021). Spanish language models.

Hasan, M. R., Maliha, M., and Arifuzzaman, M. (2019). Sentiment analysis with nlp on twitter data. In 2019 International Conference on Computer, Communication, Chemical, Materials and Electronic Engineering (IC4ME2), pages 1-4.

Isaak, J. and Hanna, M. J. (2018). User Data Privacy: Facebook, Cambridge Analytica, and Privacy Protection. Computer, 51(8):56-59. Conference Name: Computer.

Kamal, O., Kumar, A., and Vaidhya, T. (2021). Hostility Detection in Hindi leveraging Pre-Trained Language Models. arXiv:2101.05494 [cs]. arXiv: 2101.05494.

Kandias, M., Stavrou, V., Bozovic, N., Mitrou, L., and Gritzalis, D. (2013). Can We Trust This User? Predicting Insider's Attitude via YouTube Usage Profiling. In 2013 IEEE 10th International Conference on Ubiquitous Intelligence and Computing and 2013 IEEE 10th International Conference on Autonomic and Trusted Computing, pages 347-354.

Konstantinidis, K., Papadopoulos, S., and Kompatsiaris, Y. (2017). Exploring Twitter communication dynamics with evolving community analysis. PeerJ Computer Science, 3:e107. Publisher: PeerJ Inc.

Kumari, K., Singh, J. P., Dwivedi, Y. K., and Rana, N. P. (2021). Bilingual Cyber-aggression detection on social media using LSTM autoencoder. Soft Computing, 25(14):8999-9012.

Lakshmi, R., Divya, S. R. B., and Valarmathi, R. (2018). Analysis of sentiment in twitter using logistic regression. International Journal of Engineering \& Technology, 7(2.33):619-621.

Nedjah, N., Santos, I., and de Macedo Mourelle, L. (2019). Sentiment analysis using convolutional neural network via word embeddings. Evolutionary Intelligence.

Paetzold, G. H., Zampieri, M., and Malmasi, S. (2019). UTFPR at SemEval-2019 task 5: Hate speech identification with recurrent neural networks. In Proceedings of the 13th International Workshop on Semantic Evaluation, pages 519-523, Minneapolis, Minnesota, USA. Association for Computational Linguistics.

Pereira-Kohatsu, J. C., Quijano-Sánchez, L., Liberatore, F., and Camacho-Collados, M. (2019). Detecting and monitoring hate speech in twitter. Sensors, 19(21).

Peters, M. E., Neumann, M., Iyyer, M., Gardner, M., Clark, C., Lee, K., and Zettlemoyer, L. (2018). Deep contextualized word representations. cite arxiv:1802.05365Comment: NAACL 2018. Originally posted to openreview 27 Oct 2017. v2 updated for NAACL camera ready.

Pitropakis, N., Kokot, K., Gkatzia, D., Ludwiniak, R., Mylonas, A., and Kandias, M. (2020). Monitoring Users' Behavior: Anti-Immigration Speech Detection on Twitter. Machine Learning and Knowledge Extraction, 2(3):192-215. Number: 3 Publisher: Multidisciplinary Digital Publishing Institute.

Plaza-Del-Arco, F. M., Molina-González, M. D., Ureña-López, L. A., and Martín-Valdivia, M. T. (2020). Detecting Misogyny and Xenophobia in Spanish Tweets Using Language Technologies. ACM Transac- 
tions on Internet Technology, 20(2).

Pomares-Quimbaya, A., López-Úbeda, P., and Schulz, S. (2021). Transfer Learning for Classifying Spanish and English Text by Clinical Specialties. Studies in Health Technology and Informatics, 281:377-381.

Rodríguez Maeso, S. (2018). "Europe" and the Narrative of the "True Racist": (Un-)thinking AntiDiscrimination Law through Race. Oñati Socio-legal Series, 8(6):845-873.

Roesslein, J. (2020). Tweepy: Twitter for python! URL: https://github.com/tweepy/tweepy.

Roy, P. K., Tripathy, A. K., Das, T. K., and Gao, X. (2020). A framework for hate speech detection using deep convolutional neural network. IEEE Access, 8:204951-204962.

Saha, B. N., Senapati, A., and Mahajan, A. (2020). LSTM based Deep RNN Architecture for Election Sentiment Analysis from Bengali Newspaper. In 2020 International Conference on Computational Performance Evaluation (ComPE), pages 564-569.

Sany, M. M. H., Keya, M., Khushbu, S. A., Rabby, A. S. A., and Masum, A. K. M. (2022). An Opinion Mining of Text in COVID-19 Issues along with Comparative Study in ML, BERT \& RNN. arXiv:2201.02119 [cs]. arXiv: 2201.02119.

Sayan, P. (2019). Enforcement of the anti-Racism legislation of the European Union against antigypsyism. Ethnic and Racial Studies, 42(5):763-781.

Sazzed, S. (2021). Identifying vulgarity in Bengali social media textual content. PeerJ Computer Science, 7:e665. Publisher: PeerJ Inc.

Shahri, M. P., Lyon, K., Schearer, J., and Kahanda, I. (2020). DeepPPPred: An Ensemble of BERT, CNN, and RNN for Classifying Co-mentions of Proteins and Phenotypes. Technical report. Company: Cold Spring Harbor Laboratory Distributor: Cold Spring Harbor Laboratory Label: Cold Spring Harbor Laboratory Section: New Results Type: article.

Sharma, A., Kabra, A., and Jain, M. (2022). Ceasing hate with MoH: Hate Speech Detection in Hindi-English code-switched language. Information Processing \& Management, 59(1):102760.

Sun, C., Qiu, X., Xu, Y., and Huang, X. (2019). How to fine-tune bert for text classification? In Sun, M., Huang, X., Ji, H., Liu, Z., and Liu, Y., editors, Chinese Computational Linguistics, pages 194-206, Cham. Springer International Publishing.

Talita, A. and Wiguna, A. (2019). Implementasi algoritma long short-term memory (lstm) untuk mendeteksi ujaran kebencian (hate speech) pada kasus pilpres 2019. MATRIK : Jurnal Manajemen, Teknik Informatika dan Rekayasa Komputer, 19(1):37-44.

Travis, A. (2017). Anti-muslim hate crime surges after manchester and london bridge. The Guardian.

Vaswani, A., Shazeer, N., Parmar, N., Uszkoreit, J., Jones, L., Gomez, A. N., Kaiser, L. u., and Polosukhin, I. (2017). Attention is all you need. In Guyon, I., Luxburg, U. V., Bengio, S., Wallach, H., Fergus, R., Vishwanathan, S., and Garnett, R., editors, Advances in Neural Information Processing Systems, volume 30. Curran Associates, Inc.

Velankar, A., Patil, H., Gore, A., Salunke, S., and Joshi, R. (2021). Hate and Offensive Speech Detection in Hindi and Marathi. arXiv:2110.12200 [cs]. arXiv: 2110.12200.

Vrysis, L., Vryzas, N., Kotsakis, R., Saridou, T., Matsiola, M., Veglis, A., Arcila-Calderón, C., and Dimoulas, C. (2021). A web interface for analyzing hate speech. Future Internet, 13(3).

Watanabe, H., Bouazizi, M., and Ohtsuki, T. (2018). Hate Speech on Twitter: A Pragmatic Approach to Collect Hateful and Offensive Expressions and Perform Hate Speech Detection. IEEE Access, 6:13825-13835.

Zhang, Y., Fu, Z., Huang, F., and Liu, Y. (2021). Pmmn: Pre-trained multi-modal network for scene text recognition. Pattern Recognition Letters, 151:103-111.

Zhang, Z. and Luo, L. (2019). Hate speech detection: A solved problem? The challenging case of long tail on Twitter. Semantic Web, 10(5):925-945.

Zhao, J., Zeng, D., Xiao, Y., Che, L., and Wang, M. (2020). User personality prediction based on topic preference and sentiment analysis using 1stm model. Pattern Recognition Letters, 138:397-402. 